\title{
Abordaje del exceso de peso en población pediátrica, más allá de una intervención netamente clínica
}

\section{Al Editor:}

A nivel global las patologías cardiometabólicas siempre han sido materia de discusión en salud pública, la aparición de casos nuevos dificulta el control de la enfermedad en la población. La prevalencia de la obesidad entre niños, niñas y adolescentes en comparación con años anteriores muestran un incremento en el $47 \%$, teniendo esto un impacto negativo a corto y largo plazo en todos los ámbitos referentes al bienestar físico, psicológico, social y económico (1).

En Colombia, el exceso de peso en los menores en edad escolar se incrementó del 18,8\% en 2010 a 24,4\% en 2015, según demuestran los datos de la Encuesta Nacional de Situación Nutricional (ENSIN) (2), lo que denota la importancia de implementar medidas oportunas en nuestro territorio nacional para disminuir la incidencia de la enfermedad.

Dentro de las intervenciones que han venido en auge con respecto a la prevención de la obesidad infantil, se resaltan las intervenciones basadas en la familia, dado que en varios estudios e informes, los padres se ven como punto clave para el manejo de los comportamientos que afectan el consumo y gasto de energía en los niños (dieta, actividad física, uso de medios y sueño) (1), no obstante, las intervenciones se han centrado principalmente en las dos primeras, descuidando los últimos dos predictores.

Resulta curioso que en el mismo gremio médico se ha infravalorado este tema, dado que solo se realizan recomendaciones superficiales sobre alimentación, dejando a un lado todo un campo que deriva de la obesidad infantil. Entender este campo permitirá intervenir adecuadamente, evitando las numerosas complicaciones que derivan de esta patología. La evidencia relaciona la obesidad infantil con enfermedades crónicas (por ejemplo, el asma y la diabetes mellitus tipo II), factores de riesgo cardiovascular (por ejemplo, la hipertensión arterial) y alteraciones en la salud mental (por ejemplo, la baja autoestima), entre otras. Adicionalmente, el niño que permanece con sobrepeso $\mathrm{u}$ obesidad hasta la adultez aumenta el riesgo de morbilidad de por vida y la mortalidad prematura (3).

Este aumento de la morbimortalidad está ligado con el aumento del riesgo cardiovascular, ya que presentan un sistema renina angiotensina aldosterona patológicamente activo, lo que trae consecuencias de vasoconstricción a nivel renal. A su vez, existe un desbalance entre el sistema simpático y parasimpático, lo que aumenta la cantidad de catecolaminas circulantes, dando como resultado un aumento de la presión arterial y finalmente la presencia de un estado proinflamatorio crónico, que propicia el deterioro progresivo de órganos y tejidos (4).

Dadas las consecuencias de esta enfermedad, para su manejo se han priorizado varias intervenciones en las áreas de prevención y tratamiento, dentro de las cuales se resaltan: la realización de mínimo 60 minutos diarios de actividad física de intensidad moderada a vigorosa (incluidos juegos); la participación en talleres educativos sobre alimentación saludable; la vinculación de cambios del entorno (por ejemplo, ofrecer agua en la cafetería o en la tienda escolar); las intervenciones de detección y atención temprana (por ejemplo, detección de sobrepeso u obesidad en estudiantes por parte de enfermeras escolares); y las mejoras en el servicio de alimentación escolar (por ejemplo, ofertados menús saludables y culturalmente aceptables) (3). Adicionalmente es útil valerse de campañas en los medios de comunicación (5).

Frente a la regulación del etiquetado de productos procesados y ultraprocesados, se ha llegado a estimar que esta medida podría influir en las respuestas de la industria, generando reformulaciones en sus productos, así como también podría influir en la compra y consumo de estos. Bajo esta premisa, algunos países de Latinoamérica y el mundo ya han implementado la medida. En una revisión sistemática con metaanálisis que incluyó 60 estudios a nivel mundial, se determinó que el etiquetado de alimentos redujo el consumo de calorías y grasas, al tiempo que aumentó el consumo de verduras, según este estudio también se encontró que el etiquetado de los alimentos alteraba las formulaciones de la industria, disminuyendo el contenido de sodio y grasas trans en sus productos (5).

Esta iniciativa podría ser potencialmente útil en la reducción del exceso de peso, teniendo en cuenta las dificultades que ha presentado el consumidor para poder tomar decisiones informadas frente a lo que va a consumir o no. Los productos ultraprocesados se tienden a caracterizar por un alto contenido de azúcares, grasas saturadas, energía y sodio, contenidos que suelen desconocerse por la dificultad en la lectura de las etiquetas actuales.

Otros enfoques complementarios pueden ser cruciales para combatir el exceso de peso en niños, es relevante generar procesos de información, educación y comunicación valiéndose de las nuevas tecnologías y de las campañas en los medios de comunicación (5).

Sin duda alguna, el abordaje del exceso de peso es también una cuestión política en constante evolución, pese a que continúan existiendo múltiples dudas con respecto a su manejo, sobretodo en la edad pediátrica, se considera que es una

Este es un artículo de acceso abierto distribuido bajo los términos de la licencia Creative Commons Attribution-NonCommercial-NoDerivs 3.0 IGO, que permite su uso, distribución y reproducción en cualquier medio, siempre que el trabajo original se cite de la manera adecuada. No se permiten modificaciones a los articulos ni su uso comercial. Al reproducir un articulo no debe haber ningún indicio de que la OPS o el artículo avalan a una organización o un producto específico. El uso del logo de la OPS no está permitido. Esta leyenda debe conservarse, junto con la URL original del artículo. Crédito del logo y texto open access: PLoS, bajo licencia Creative Commons Attribution-Share Alike 3.0 Unported. 
problemática que requiere una o varias intervenciones eficientes y costo-efectivas, siendo implementadas de manera oportuna, sobre todo teniendo en cuenta que la población que se encuentra en mayor riesgo de presentar la enfermedad, es aquella que presenta mayores brechas de inequidad, personas de los estratos socioeconómicos y niveles educativos más bajos o ubicadas en áreas rurales.

En este sentido, se realiza un llamado a la academia, la industria, el sector educativo y de la salud, así como a la comunidad en general, para tomar acciones mancomunadas que permitan abordar de manera integral una patología creciente en la población infantil, reconociendo que el exceso de peso se da como resultado de múltiples factores, los cuales son poco abordados en la práctica clínica y en las medidas de salud pública tomadas en la actualidad.

Conflicto de intereses. Ninguno declarado.
Declaración. Las opiniones expresadas en este manuscrito son responsabilidad de los autores y no reflejan necesariamente los criterios ni la política de la RPSP/PAJPH y/o de la OPS.

\section{Lucía Tatiana Romero Pineda \\ Nelson José Villarraga Marquez \\ Sebastián Jurado Bastidas}

Universidad de La Sabana. Bogotá, Colombia. $₫$ Lucía Tatiana Romero Pineda, luciaromerop96@gmail.com

\section{Jenny Paola Pachón Romero}

Universidad Nacional de Colombia. Bogotá, Colombia

Forma de Citar Romero Pineda LT, Villarraga Marquez NJ, Jurado Bastidas S, y Pachón Romero JP. Abordaje del exceso de peso en población pediátrica, más allá de una intervención netamente clínica. Rev Panam Salud Publica. 2021;45:e132. https://doi.org/10.26633/ RPSP.2021.132

\section{REFERENCIAS}

1. Ng M, Fleming T, Robinson M, Thomson B, Graetz N, Margono $\mathrm{C}$ et al. Global, regional, and national prevalence of overweight and obesity in children and adults during 1980-2013: a systematic analysis for the Global Burden of Disease Study 2013. The Lancet. 2014;384(9945):766-781.

2. ENSIN: Encuesta Nacional de Situación Nutricional [Internet]. Instituto Colombiano de Bienestar Familiar. 2021. Disponible en: https://www.icbf.gov.co/bienestar/nutricion/encuesta-nacionalsituacion-nutricional\#ensin3. Acceso el 26 de febrero 2021

3. Bleich SN, Vercammen KA, Zatz LY, Frelier JM, Ebbeling CB, Peeters A. Interventions to prevent global childhood overweight and obesity: a systematic review. Lancet Diabetes Endocrinol. 2018;6(4):332-346. doi: 10.1016/S2213-8587(17)30358-3
4. Chrysant S. Pathophysiology and treatment of obesity-related hypertension. The Journal of Clinical Hypertension. 2019;21(5):555-559

5. Shangguan S, Afshin A, Shulkin M, Ma W, Marsden D, Smith J, et al. A Meta-analysis of Food Labeling Effects on Consumer Diet Behaviors and Industry Practices. 56. 2019;2:300-14.

Manuscrito recibido el 12 de julio de 2021. Aceptado para publicación el 17 de agosto de 2021. Sin arbitraje externo. 